\title{
A Multi-Agent Based Approach to Short Message Service (SMS) Normalization System
}

\section{Ademola O. Adesina}

Department of Mathematical Sciences

(Computer Science Unit)

Faculty of Sciences

Olabisi Onabanjo University

Ago-Iwoye, Nigeria

\section{Correspondence}

Adesina Ademola Olusola,

Department of Mathematical Sciences (Computer Science Unit),

Faculty of Science,

Olabisi Onabanjo University,

Ago-Iwoye, Nigeria

Email:inadesina@gmail.com

\begin{abstract}
:
Introduction: SMS language is characterized with fashion and user's creativity but needs transformation to proper English words or spelling to formulate natural language and text processing activities.

Aims: The proposed application, Web Information Retrieval System Architecture Based on SMS (WIRSABoSMS), normalizes SMS language to retain its original syntactic structure.

Material and Methods: The concept of mobile agents in web technology was introduced as a medium to achieving Short Message Service (SMS) normalization. SMS normalization was carried out with the adoption of multi agent technology, as agents are involved in character search, sort, and compare of the strings written in SMS form into its parental orthography. This architecture was designed based on web information retrieval system (IRS) in order to achieve SMS normalization. BLEU (bilingual evaluation understudy) was used to evaluate the quality of text. BLEU scores compare the human judgment with that of the machine translation using two set of corpora.
\end{abstract}

Results: The outcome of syntactic text message normalization recorded an average of $90 \%$ performance (for the corpus collected from researchers) when compared with the similar test conducted with human judgment using BLEU scores metric in an health related domain.

Conclusion: This study has been able to adopt a mixed technique of both multi-agents and Web Information Retrieval System (known as WIRSABoSMS) to address SMS normalization. The technique involves Language Modelling (LM) as it does not involve SMS corpus, which is very scarce. Future plans involve using the technique in more challenging technologies by applying it to set of queries in search engine, question and answering system (QAS), and frequently asked questions (FAQ) scenario. 


\section{INTRODUCTION}

The advancement in today's technology has permitted data transmission in Web and Mobile communications as data are transferred in form of voice, text, image signals, within and outside different communication platforms thus enhancing interoperability. Web technology has opened many opportunities for online activities including e-commerce based transactions [1] population information system or population detection [2-3], development of content for learning technology systems [4-5], medical application [6-7], semantic web [8-9] etc. The perfect communication advantage in Web and Mobile connections and services have given room for better integration and management of different targeted consumers as interoperability among different web contents and users are enhanced. This had previously not been the case, as activities were managed separately [8] before web technology came into existence.

A noticeable interoperability advantage in the mixed technologies of Web and Mobile communications is the invention of short message service (SMS) communication, also referred to as text messaging. Text messaging has become ubiquitous as it is useful wherever mobile phone communication or services are available. For many users, mobile phones function first as text messaging devices, and secondly as voice calling devices [10-11]. SMS communication has significant challenges because of the noisy nature and unstructured format in which the language is put or used. Text messages are associated with non-formal writing styles, including colloquialism, misspellings and homophonic abbreviations. This needs to be parsed into formal language, for example English, for it to be amenable to information search retrieval [12-13] and other text processing works under natural language processing.

On one hand, the informal technique of writing the language creates difficulty in using it as a query in a search engine in Information Retrieval System (IRS) and on the other hand, the concern in the research field of IRS is the user's needs and satisfaction when results are returned for every SMS query. There is no system that can return hundred percent relevant documents in response to users' query. An ideal retrieval system is expected to retrieve only relevant documents and no irrelevant documents. However, this is almost impossible as perfect retrieval systems do not exist, and will not exist because search statements are necessarily incomplete and the relevance depends on the subjective view of the users [14-15]. With the ingenuity of the SMS language and its writing, it is certain that search engine will treat the language as garbage, this establishes the long time slogan of Computer Science that says: Garbage-InLASU Journal of Research and Review in Science
Garbage-Out (GIGO). SMS naturally lacks the functionality to search, collect and aggregate information from the search engine for any user on the fly or move.

There is a need to normalize SMS to be able to serve the role of query terms in IRS environment using the Web as the platform for accessing information. It is on this note that the research on designing a Web Information Retrieval System Architecture Based on SMS Normalization (WIRSABoSMS) was carried out. The architecture relies on multi-agents platform to make the necessary normalization to SMS terms in form of spelling corrections, insertion of missing letters, replacement of homophones, substitution of numbers with letters, recognition of abbreviations and re-fixing of punctuations through different components that constitute the architecture of the design. Meanwhile, web portals provide a good solution for collecting relevant information from and for the user, but the defect is that it lacks users personalization and centralized architecture [16] because it serves as gateway for SMS communication.

\subsection{WEB PORTALS AND SMS COMMUNICATION}

The emergence of the web has brought about the development of new information retrieval techniques such as peer-to-peer information retrieval, multimedia information retrieval, semantic web retrieval system [17-18], and web information retrieval techniques [16, 19-20] in which hyper-link analysis and the use of anchor text are the most obvious, but spam detection, page quality assessment, URL analysis, and a multitude of other features have become important [21. 22]. The problem with web information retrieval system is with the dynamism of the web, its rate of growth and change, and the increasing richness and complexity of the information sources available in it [18, 23]. As good as most of these technologies and the information retrieval method systems are, little or no research has been considered in the literature review on the use of SMS to make enquiries from the web search engine. Therefore SMS normalization using the advantages of Web and Mobile technologies to enhance information retrieval for people using cell-phones as a medium of information communication was proposed. SMS as a means of communication features includes the use of small keyboards, sharing one-to-one, many-to-one or one-to-many relationship in broadcast. It adopts new habits of writing i.e. new abbreviations inventions, nonstandard orthographic forms that are assembled together to make meaning between or among the users faster with fewer words or characters, commonly among the youth [10, 24].

Web portals stand as a gateway [25-26] for easy access to the depository and provision of other web 
resources for web users. Web portals help organizations, professionals and communities easily disseminate information, effectively share knowledge, and creatively engage in collaborative endeavours [20, 27]. Web portal is defined as a web site for specific audience that aggregates an array of content and provides a variety of services like search engines, directories, news, e-mail and chat rooms [25-26]. The Portal provides a full range of knowledge management and collaboration services through the posting of key documents, events, press releases, discussion forums, minutes of meetings, points of contact, and other relevant information [25-26]. The web has remained the only vehicle to get information not only to external constituents but could also be extremely useful to communicate procedures, common documents to internal constituents.

Web portals are built for several reasons, as they are managed differently from traditional IRS and they share different architectures. It may involve semantic Web, Web services, and multi-agent technologies to enable not only precise location of Web resources but also retrieve automatically the integration of hybrid web contents and its services [16]. Web services promote avenues for application interoperability between and within heterogeneous environments. The available web-based and object-oriented technologies are created for developing and supporting e-commerce services in business environments like procurement, billing, accounting, human resources and other services. As new infrastructures is a challenge in achieving a higher degree of intelligence and automation of e-services $[1,28]$

Using Peer-To-Peer (P2P) computing architecture connection in the web portal built for SMS normalization, the proposed architecture, (WIRSABoSMS), has directed emphasis on the efficient method of communication with the aim of reducing communication load. In $\mathrm{P} 2 \mathrm{P}$, the nodes are independently computing and can collaborate within the system if the need be so as to achieve a common goal $[6,8]$. The P2P architectural systems are always robust, scalable and cheap to maintain, when they are decentralized from other architecture and this enhances the advantage of having large amount of information transferred between peers [8]. Therefore an efficient mechanism of reducing communication load without compromising the level of recall and precision from the information retrieved in the $\mathrm{P} 2 \mathrm{P}$ architectural model is paramount in the design. There is therefore a need to involve a multi-agent based communication system in the web IRS so as to connect, create, query, browse and manage the proposed SMS normalization model.

It is important to provide a gateway i.e. web portal [2526] in order to access the web-server for SMS normalization because of the frequent user's requests. The information can be made available at local or remote levels. Information related to the users' profile (contacts, emails) is made available at the local level, whereas information that relates to publishing is handled at the remote side by the experts. Such information are always searched and accessed through the Web application, such as WIRSABoSMS, as there will be a need for users to access, therefore gateway is very important in this situation. Web portal serves a very important role in the proposed architectural design i.e. WIRSABoSMS, as it serves as a medium in correcting abnormalities in SMS writing.

\subsection{MULTI-AGENT SYSTEMS}

A multi agent system involves a decentralized computing approach to allocate and schedule tasks on a massively distributed grid. The potential properties of the multi-agent systems, dynamically creates and dissociates clusters to serve the changing resource demands of a global task queue [29]. Multi-agent systems use distributed agents to either model or solve a problem. An agent is an entity which matches some real-world object. The agents move about in space and interact with other agents in their neighbour-hood according to the encoded rules. Agents specification that are responsible for Control, Distributed Energy Resources (DER), User and Database in a IDAPS Intelligent Distributed Autonomous Power Systems multi-agent system was designed and implemented using multi-agent technology in the context of IDAPS. The agents in the IDAPS multi-agent system work in collaboration to detect upstream outages and react accordingly to allow the micro-grid to operate autonomously in an island [30].

Various architectural properties of multi-agent systems were discussed in the report of Shehory [31] with emphasis on architectural characteristics. Even though differences in their designs and implementations result in variations in their strengths and weaknesses addressing computational problems, properties, class of problems that can be developed or modified. Among the cases discussed are: the ARCHON (an architecture for multi-agent systems) infrastructure; OSACA (an Open System for Asynchronous Cognitive Agents) and DIDE (a Distributed Intelligent Design Environment) infrastructure multi agent infrastructure; ADEPT (Advanced Decision Environment for Process Tasks) infrastructure; DESIRE (DEsign and Specification of Interacting REasoning components) architecture; MACRON (Multi-agent Architecture for Cooperative Retrieval ONline) infrastructure, Federated MAS (multi-agent systems); The RETSINA (Reusable Task Structure based Intelligent Network Agents) Multi-Agent Infrastructure and the RESTINA Agent internal Architecture [31]. 
Based on the assessment of the MAS community, agents have the following properties

- Autonomy. An agent possesses individual goals, resources and competences; as such it operates without direct human or other intervention, and has some degree of control over its actions and its internal state.

- Adaptability. An agent has the control over its own state and so can regulate its own functioning without outside assistance or supervision.

- Sociability. An agent can interact with other agents, and possibly humans, via some kind of agent communication language. Through this means, an agent is able to provide and ask for services.

- Reactivity. An agent perceives and acts, to some degree, on its close environment; it can respond in a timely fashion to changes that occur around it.

- Pro-activeness. Although some agents, called reactive agents, will simply act in response to stimulations from their environment, an agent may be able to exhibit goal-directed behaviour by taking the initiative. [32-34]:

\section{PROPOSED ARCHITECTURE BASED ON MULTI-AGENT APPROACH FOR SMS NORM ALIZATION - WIRSABOSMS}

Figure 1, depicts the overview of the SMS normalization architecture showing various components and the agents involved in the design of WIRSABoSMS. The conceptual approach involves a multi-agent system acting in form of search, display, login, evaluation, input, output and are being summarized under the key components of the architectural technique and the conceptualization approach to WIRSABoSMS normalization framework. It is important to note that the architectural design establishes a well-defined modularization technique for SMS normalization that involves the machine learning approach under multi- agent based environment. The following advantages of multi-agent technology were used: faster problem solving using exploiting parallelism within the multi-agent based configuration; decreasing communication in high level transmissions under transmission of high-level partial solutions to other agents rather than raw SMS data; team work among other agents in solving a current SMS normalization problem; and improvement in reliable and fault tolerance with collaboration of other agents within the multi-agent environment, in case there is a failure on the path of one of the multi agents.

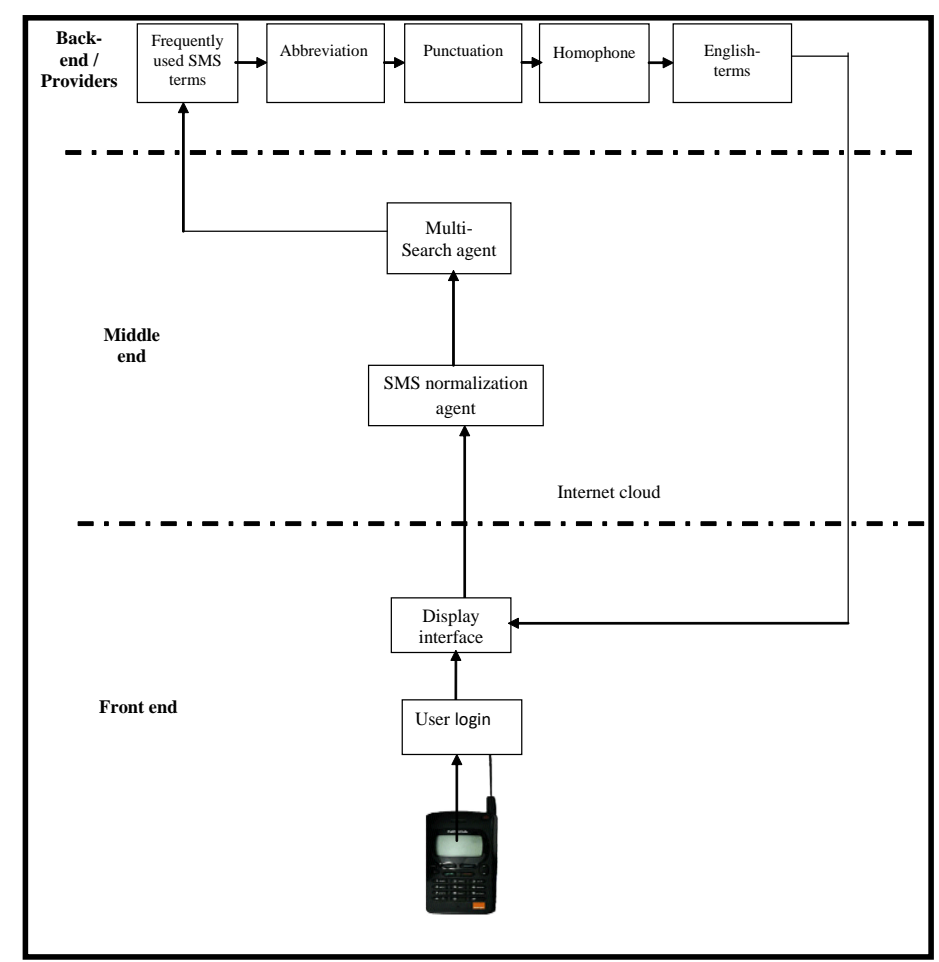

Figure 1: Overview of the SMS normalization architecture showing various components and the agents involved

Web portal links the user to his or her information needs in the web-server. The proposed SMS normalization web-based application has features to reformulate the query in case the expected or emerging results do not satisfy the user. The ultimate goal of the architecture is to return appropriate results as an answer or a set of answers from the information made available in the Question-Answer and Frequent Asked Question repository using SMS as a source of information query. The portal serves the duo roles of communication between the users' interface (Frontend) and web server (Back-end). In the front end, the browser links the user to the SMS normalization architecture/algorithms. The front-end provides the users with the SMS normalization browsers. The browser and SMS-normalization search engine functionalities perform actively on the Englishalphabet, homophone, abbreviation, preposition and punctuation components.

\subsection{WIRSABOSMS-ARCHITECTURE ALGORITHM}

The typical WIRSABoSMS algorithm steps are shown: Step 1: Log in and confirm the security through login agent

Step 2: Input Text (T) as SMS Message

Step 3: Search each character $c$ in the corresponding database $(A)$

Step 4: Begin

Step 5: SMS input text T=t1 t2: : : tn; where the $\mathrm{t} 1$ for $i \in[1 \ldots . . n]$ are tokens

delimited by spaces or punctuation marks such as ., :, ;, ?, =, @, etc. 
Step 6: Token $t 1 \in T$ is in turn organized into its component sequence of individual characters $\mathrm{tj}=[\mathrm{ci} 1, \mathrm{ci} 2 \ldots \mathrm{cmi}]$

Step 7: Foreach child c(i) of Text(T)

Step 8: Search (A(i))

Step 9: $\mathrm{T}: \mathrm{t}+1$

Step 10: $A(T):=$ Found from the Module in the corresponding order Solution(A) then Go To Step 12 i.e. Report solution

\section{Otherwise}

Step11: Reformulate in Step 2 Endfor

Step12: End

\subsection{FUNCTIONALITY OF THE WIRSABOSMS ARCHITECTURE}

The functionality of the architecture (WIRSABoSMS) is based on a multi-agent collaborative system that manages the normalization of the SMS vis-à-vis the search technique in the English-alphabet, homophone, abbreviation, preposition and punctuation components. The architecture is divided into three components: namely front-end, middle-end and the back-end.

\subsubsection{Front End}

This links the user to all the information s/he needs. It is responsible for initiating the SMS communication between the user and the WIRSABoSMS application. The user has influence over this part of design, in terms of login, display of the input and output, as well as query reformulation. This resides on the handset; it is designed to satisfy users' personal information and model. Given the wide range of handsets in circulation - from the iPhone, Symbia to touch screen - and each with its different keystroke, users are expected to have a working knowledge of their individual handsets, but not necessary be concerned with the technology behind the construction of the cell-phone. It is important to note that the front end serves as the browser link to the web server. Its function extends to accessing platforms for all local users from web information accessing to other web sites, as the conventional browser.

The Front end performs the role of user's login ( $\mathrm{UI})$, display interface (DI) and query management (QM). DI and $\mathrm{Ul}$ agents play active roles in taking the input into the middle layer and showing the results of the process after the SMS normalization and parsing. Users use the log-in to gain access to and exit from the web application (WIRSABoSMS) - this is controlled by the log-in agent. Front end represents the beginning of the process of web based SMS normalization. It is open to the users; it has the user name and the corresponding password. Both old and new SMS users will have to login to the system. The system is built such that, the web application is denied to users whose security details do not reside in the web server. LASU Journal of Research and Review in Science
The login agent fetches the data from the login table in the database and access is denied where the user's name and password record does not correspond with details entered by the user; there must be a match to gain access to the web application.

One other major role played by the $\mathrm{Dl}$ is displaying the result of the processes after SMS normalization and parsing. DI agent fetches the results from the SMS normalization agent and displays it on the screen for the user to decide upon the outcome. As with the input process, the $\mathrm{Dl}$ agent takes the enquiry (data) entered by the user or the response expected by the application to the processing layer for further processing. The connect button is a link to the processing stage i.e. the web server and the web application, WIRSABoSMS.

The unique learning operation known as query management is performed at the front end, as query agent history (QAH) is expected to have a collaborative effect with the user model. This is very crucial because sometime, individual agents may not be skilled or knowledge-able enough to return an answer to request. The collaborative agent in the Query/search agent history gradually creates virtual agent communities, where agents with the same interest and patterns stay together. There may be no need to go beyond this point, if the request is made available or present. This reduces communication load in the architecture. The higher the number of queries inputted, the more the agent acquires links to the new knowledge. The collaborative agent in the UI employs three types of agents: User Interface (UI) or Display Interface (DI) agent, Information retrieval (IR) agent from the SMS normalization agent (SMSNA) and History Management (HM) agent. The set of agents $\mathrm{UI} / \mathrm{DA}, \mathrm{IR}$ and HM are assigned to each SMS user and these serve as the user's profile - this is where the learning technique of the architecture stands.

\subsubsection{Middle End}

This layer conveys the resources or requests from the front end. The middle end, otherwise known as the middle layer or mediator level, enables communication between the front end and back end. The front end submits SMS queries based on the user's requirement for web searching to the provider. The back-end responds to the query by providing the appropriate results based on the need of the users. The middle layer is a multi-agent based system assigned to the need of the users' requirement using a P2P information retrieval model for satisfying the search and access tasks.

The communication between the front-end and backend can be referred to as a multi-agent peer-to-peer (MAP2P) system that uses the function of various 
search agents and data tables to perform the needs in the back end. The major assignment of the search agents in the back-end is to search and exchange the users' needs at both (front and back) ends of the architecture. The block is collectively referred to as SMS search normalization agent (SMSSNA) as it works out the particular table which the agent searches for on the web resources or a provider that has the resources as the intermediary.

Between and within the components and architecture are sufficient communication agents that execute transfer of web resource instructions during SMS query or reformulation in the WIRSABoSMS architectural system.

\subsubsection{Back End}

Back end is assigned to transport a variety of information that satisfies the SMS request sent by the users. It sources this from different components that constitute the block (Figure 2). The components are designed according to the alphabets, figures, punctuation marks present in English language. For instance, English alphabet component has 26 letters (A - Z or $\mathrm{a}-\mathrm{Z}$ ) and therefore 26 tables are created according to the alphabet; homophones component has additional figures besides the alphabets to give room for words that users include figures, that is, alphanumeric (e.g. 2gether, gr8, 2nite, 4ever, 8ty etc.)

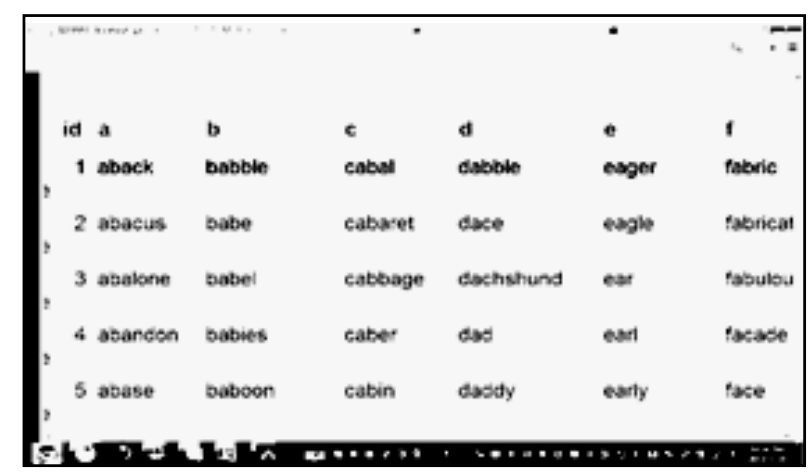

Figure 2: Web database server

Abbreviation and preposition components all have letters in their tabular form and this stands for all manners of words in abbreviation e.g. AIDS, DNA, HIV, USA occupying $A, D, H, \cup$ Tables respectively. It is the assigned agents that execute the information retrieval after searching tasks from the components within and between the components in the back-end or provider level. These activities are performed at the web-servers when the SMS normalization (WIRSABoSMS) was implemented.

\subsection{METHODOLOGY}

The SMS requests are presented in input form that conveys the query. The form requests the user to "Ask a question" and the command button "Ask" makes the interface to the SMS normalization algorithm. When the command is issued, it is parsed into the database (Figure 3). The database contains over forty thousand data within the repository spreading from English words, homophones, abbreviations, acronyms to prepositions structured according to the alphabets (for English words, abbreviations, acronyms and prepositions) and alphabets and figures for homophones.

This processing phase serves duo purposes, first taking in the request from the SMS users and secondly giving out the results of the enquiries. The result in the snapshot (Figure 3) shows an SMS word abndn which is sent and returned an answer abandon. The process involves confirming if the word exists in the dictionary, if it does, the result is returned, otherwise it looks for it in all other tables and components until it is found. In the worst case scenario - where it is not found - then it becomes a new word for the architecture to learn. The Learning Agent (LA) can request the option of keeping the word in the database and it is kept on the user's profile. This is one important way in which the architecture learns new words and increases the volume of words in the dictionary.

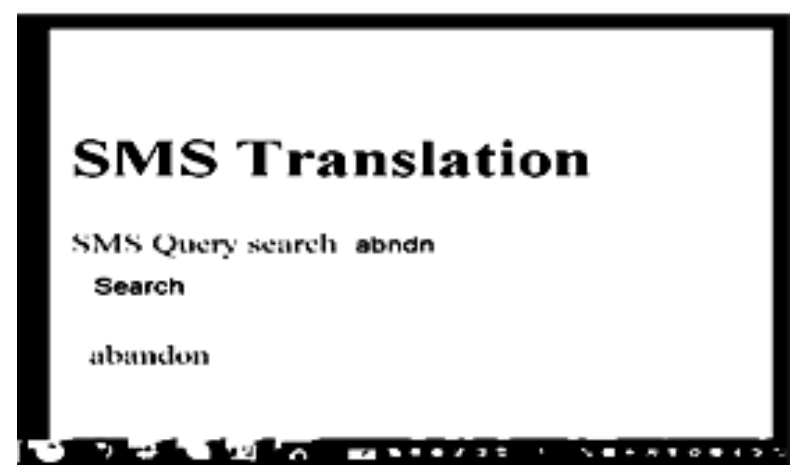

Figure 3: Query and Results phase

Another possible scenario may arise where the number of results after parsing is greater than one, depending on the number of words that are matched in the database. The user is given the option of choosing which of the words s/he originally wished to parse.

\section{EVALUATION AND RESULTS}

Further evaluation was carried out based on BLEU score [35-36]. BLEU scores compare the human judgment with that of the machine translation using two set of corpora from Caroline [37] and Liu [38]. These two sets of corpora were requested from the researchers in the course of the experiment. Upon downloading the text into Microsoft text editor brought out the following messages:

there are too many spelling or grammatical errors in the SMS set, to continue displaying them. To check the spelling and grammar of this document, choose Spelling and Grammar from the Review tab 
this message is a characteristic feature and evidence of SMS. It shows the extent of creativity in the corpus. Table 1 shows the sample of SMS words in the corpus collected for this experiment.

Table 1: Samples of SMS words in our corpus

\begin{tabular}{|l|l|}
\hline SMS example & Normalization \\
\hline cya & see you \\
\hline tomoz & Tomorrows \\
\hline numba & Number \\
\hline prez & Present \\
\hline ursef & Yourself \\
\hline orite & Alright \\
\hline
\end{tabular}

Table 2 shows an overall improvement in the normalized SMS from its Original SMS text judging from the number of words in the corpus, average number of words per message length, average number of characters per message length and average character per word.

Table 2. Summary of normalized SMS from Caroline Tagg [37] corpus after evaluating with WIRSABoSMS application.

\begin{tabular}{|l|l|l|}
\hline Feature & $\begin{array}{l}\text { Original } \\
\text { SMS text }\end{array}$ & $\begin{array}{l}\text { Normalized } \\
\text { SMS }\end{array}$ \\
\hline $\begin{array}{l}\text { No. of messages in the } \\
\text { corpus }\end{array}$ & 11,036 & 11,036 \\
\hline No. of words in the corpus & 190,099 & 198,500 \\
\hline $\begin{array}{l}\text { No. of SMS words in the } \\
\text { corpus }\end{array}$ & 4,012 & 3,859 \\
\hline $\begin{array}{l}\text { Average no. of words per } \\
\text { message length }\end{array}$ & 15.28 & 17.2 \\
\hline $\begin{array}{l}\text { Average no. of characters } \\
\text { per message length }\end{array}$ & 18.24 & 24.5 \\
\hline $\begin{array}{l}\text { Average character per } \\
\text { word }\end{array}$ & 4.65 & 5.7 \\
\hline
\end{tabular}

While the number of messages in the corpus remains the same, there is an improvement of $5 \%$ SMS normalization, i.e.in the number of words added to the corpus after evaluating with WIRSABoSMS. This can be interpreted or translated that some of the SMS words available in the corpus have been normalized. This is against the general rule observed from the number of tokens collected in English corpus research [39] because there are greater token in the original text than in the SMS. In trying to isolate the SMS words that were used in the corpus, our design/algorithm give $96.18 \%$ results in the normalization process with the difficulty coming up in those areas that were outside the scope of our algorithm (i.e. alphanumeric SMS). There are $13 \%, 34 \%$ and $25 \%$ improvement in the average numbers of words per message length, characters per message length and character per word respectively. A simplified setting of alignment (as a list of pair) was considered i.e. monotonic, between the source and target languages during the training and testing of the dataset. Table 3 shows the result of the normalized SMS corpus (from the researchers whose dataset were used for the experiment).

Table 3. Summary of normalized SMS from Liu corpus [38] after evaluating with WIRSABoSMS application.

\begin{tabular}{|l|l|l|}
\hline Feature & $\begin{array}{l}\text { Original } \\
\text { SMS text }\end{array}$ & $\begin{array}{l}\text { Normalize } \\
\text { d SMS }\end{array}$ \\
\hline No. of messages & 20,036 & 20,036 \\
\hline No. of words & 85,866 & 87,012 \\
\hline Characters (no space) & 216,968 & 245,325 \\
\hline Characters (with spaces) & 301,837 & 312,587 \\
\hline $\begin{array}{l}\text { No. of SMS words } \\
\text { in the corpus }\end{array}$ & 14,012 & 12,011 \\
\hline $\begin{array}{l}\text { Average no. of words } \\
\text { per message length }\end{array}$ & 3.5 & 5.6 \\
\hline $\begin{array}{l}\text { Average no. of } \\
\text { characters per } \\
\text { message length }\end{array}$ & 8.2 & 9.5 \\
\hline $\begin{array}{l}\text { Average character } \\
\text { per word }\end{array}$ & 1.8 & 3.1 \\
\hline
\end{tabular}

With the same number of messages in the corpus remains the same, there is an improvement of $2.5 \%$ SMS normalization i.e. increase in the number of words added to the corpus after evaluating with WIRSABoSMS algorithm. This can be interpreted or translated that some of the SMS words available in the 
corpus have been normalized. In trying to isolate the SMS words that were used in the corpus, our design/algorithm give $85 \%$ results in the normalization process with the difficult coming up in those areas that were outside the scope of our algorithm. There are $60 \%, 16 \%$ and $72 \%$ improvement in the average numbers of words per message length, characters per message length and character per word respectively.

\section{CONCLUSION}

This study has been able to adopt a mixed technique of both multi-agents and Web Information Retrieval System (called WIRSABoSMS) to address SMS normalization. The technique involves Language Modelling (LM) as it does not involve SMS corpus, which is very scarce. Future plans involve using the technique in more challenging technologies by applying it to set of queries in search engine, question and answering system (QAS), and frequently asked questions (FAQ) scenario. Its effectiveness may be measured in terms of precision and recall in order to measure the performance and efficiency. This method shows an in-depth knowledge of SMS normalization; from the literature review surveyed, this is the first research group that will apply web technology and multi-agent approach to SMS normalization.

\section{ACKNOWLEDGEMENT}

I appreciate Prof $O$. Folorunso for his suggestions and fruitful discussions.

\section{COMPETING INTEREST}

The author declares no conflict of interest.

\section{FUNDING}

No grant was received from any funding agency.

\section{REFERENCES}

1. Fortino, G., A. Garro, and W. Russo, E-commerce Services based on Mobile Agents, in Encyclopedia of E-Commerce, E-Government, and Mobile Commerce. 2006, IGI Global. p. 319-326.

2. Cunningham, S., et al., Using web technology to support population-based diabetes care. J Diabetes Sci Technol, Clinical Technology Centre, University of Dundee, Dundee, UK. scott.cunningham@nhs.net, 2011. 5(3): p. 523-534.

3. Oldenburg, B., et al., Using new technologies to improve the prevention and management of chronic conditions in populations. Annual review of public health, 2015. 36: p. 483-505.

4. Pahl, C. and E. Holohan, Applications of semantic web technology to support learning content development. Interdisciplinary Journal of E-Learning and Learning Objects, 2009.

5. Pástor, D., et al., New Perspectives in Instructional Design using Semantic Web Technologies: A Systematic Literature Review. 2018.

6. Aguilera, A., E. Herrera, and A. Subero. MultiAgent System for a Medical Application over Web Technology: A Working Experience. in 13th International Conference on Biomedical Engineering IFMBE Proceedings, 2009. 2009.

7. Chen, $X$, et al. Classification of Medical Consultation Text Using Mobile Agent System Based on Naïve Bayes Classifier. in International Conference on 5G for Future Wireless Networks. 2017: Springer.

8. $\mathrm{Yu}, \mathrm{H} ., \mathrm{T}$. Mine, and M. Amamiya, AgentCommunity-based P2P semantic MyPortal information retrieval system architecture. Journal of Embedded Computing, 2009. 3(1): p. 63-75.

9. Hashim, H. and S.A.M. Noah, Research Article Learning Support with Semantic Forum System. 2015.

10. Cédrick, F. and P. Sébastien. A Translated Corpus of 30,000 French SMS. in Proceedings of the $48^{\text {th }}$ Annual Meeting of the Association for Computational Linguistics. 2010 Uppsala, Sweden.

11. Cougnon, L.-A. and J.-L. Bouraoui, 8 Orality and Literacy of Telephony and SMS. Manual of Romance Languages in the Media, 2017. 23: p. 154.

12. How, Y. and M. Kan. Optimizing Predictive Text Entry for Short Message Service on Mobile Phones. in Proceedings of Human Computer Interfaces International. 2005: Mahwah, NJ: Lawrence Erlbaum Associates, Inc.

13. Canim, M., et al. A knowledge and reasoning toolkit for cognitive applications. in Proceedings of the fifth ACM/IEEE Workshop on Hot Topics in Web Systems and Technologies. 2017: ACM.

14. Maleki-Dizaji, S., Evolutionary Learning MultiAgent Based Information retrieval Systems. PhD Thesis Sheffield Hallam University, 2003.

15. Tague, R.S.A., A framework for understanding and predicting the take up and use of social networking tools in a collaborative envionment. 2016, Western Sydney University (Australia).

16. Yu, H., T. Mine, and M. Amamiya, An architecture of personal semantic web information retrieval system, in WWW 2005. 2005: Chiba, Japan. 
17. Liang, S., et al., The relevance of mobile tourism and information technology: an analysis of recent trends and future research directions. Journal of Travel \& Tourism Marketing, 2017. 34(6): p. 732-748.

18. Inkpen, D., Information Retrieval on the Internet. 2006, University of Toronto: 800 King Edward, Ottawa, ON, Canada K1N 6N5.

19. Kasemsap, K., Mastering web mining and information retrieval in the digital age. Web usage mining techniques and applications across industries, 2017: p. 1-28.

20. Staabm, S., et al., Semantic community Web portals. Computer Networks, 2000. 33 p. 473-491.

21. Webbler, W.E., Measurement in information retrieval evaluation, in Department of Computer Science and Software Engineering. 2010, The University of Melbourne. p. 1-235.

22. Samimi, P., et al., Effects of objective and subjective competence on the reliability of crowdsourced relevance judgments. Information Research: An International Electronic Journal, 2017. 22(1).

23. Gardiner, M.E., Natural language processing methods for attitudinal near-synonymy. 2013.

24. Panckhurst, R. and C. Moïse, French text messages: From SMS data collection to preliminary analysis. Lingvisticae Investigations, 2012. 35(2): p. 289-317.

25. Pienaar, H., Design and Development of an Academic Portal. International Journal of Libraries and Information Services, 2003. 53: p. 118-129.

26. Al-Rasheed, A. and J. Berri, Effective reuse and sharing of best teaching practices. Computer Applications in Engineering Education, 2017. 25(2): p. 163-178.

27. Sandkuhl, K. and H. Lehmann, Digital Transformation in Higher Education-The Role of Enterprise Architectures and Portals. Digital Enterprise Computing (DEC 2017), 2017.

28. Zhang, Y. E-Commerce Based on Agent Technology. in Advanced technology in teaching Proceedings of the 2009 3rd International Conference on Teaching and Computational Science (WTCS 2009). 2009.
29. Banerjee, S. and J.P. Hecker. A Multi-Agent System Approach to Load-Balancing and Resource Allocation for Distributed Computing. in First Complex Systems Digital Campus World E-Conference 2015. 2017: Springer.

30. Pipattanasomporn, M., H. Feroze, and S. Rahman. Multi-agent systems in a distributed smart grid: Design and implementation. in Power Systems Conference and Exposition, 2009. PSCE'09. IEEE/PES. 2009: IEEE.

31. Shehory, O.M., Architectural properties of multiagent systems. 1998: Carnegie Mellon University, The Robotics Institute.

32. Potiron, K., A.E.F. Seghrouchni, and P. Taillibert, From fault classification to fault tolerance for multiagent systems. Vol. 8. 2013: Springer.

33. Potiron, K., A.E.F. Seghrouchni, and P. Taillibert, Multi-agent system properties, in From Fault Classification to Fault Tolerance for Multi-Agent Systems. 2013, Springer. p. 5-10.

34. Ebrahimi, M., BDI Approach to Build a Single Agent of a Distributed Multi-Agent System, in Modeling and Simulation Techniques for Improved Business Processes. 2018, IGI Global. p. 24-49.

35. Papineni, k., et al., BLEU: a method for automatic evaluation of machine translation, in Proceeding of ACL. 2002. p. 311-318.

36. Awad, G., et al. Trecvid 2017: Evaluating ad-hoc and instance video search, events detection, video captioning and hyperlinking in Proceedings of TRECVID. 2017.

37. Tagg, C., A Corpus Linguistics Study of SMS Text Messaging, in Department of English. 2009, School of English, Drama and American and Canadian Studies: Birmingham. p. 402.

38. Liu, F., et al. Insertion, deletion, or substitution? normalizing text messages without pre-categorization nor supervision. in Proceedings of the 49th Annual Meeting of the Association for Computational Linguistic: Shortpapers. 2011. Portland, Oregon.

39. Pinto, D., et al., The soundex phonetic algorithm revistied for SMS-based information retrieval *. 2011 Faculty of computer science, Benemerita Universidad Autonoma de Puebla, Mexico. 